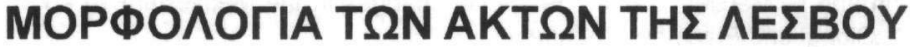

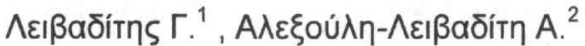

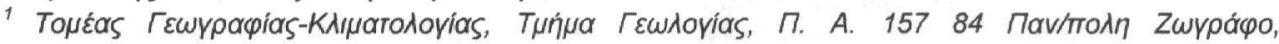 \\ livaditis@geo.uoa.gr

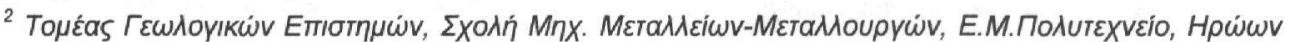

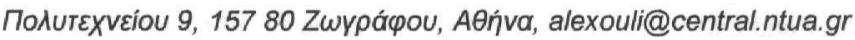

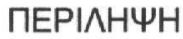

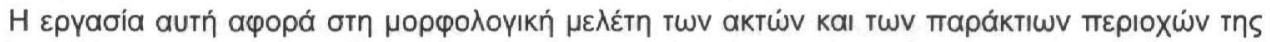

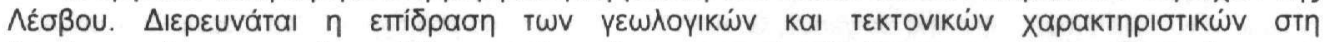

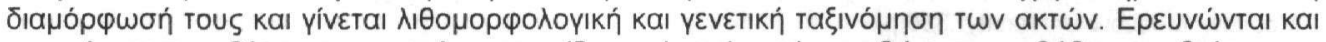

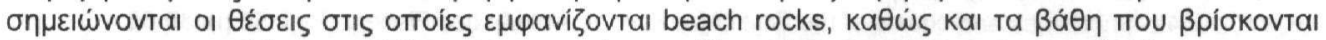

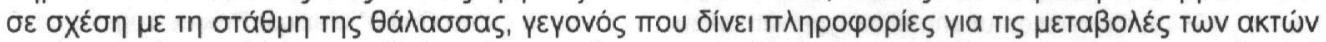

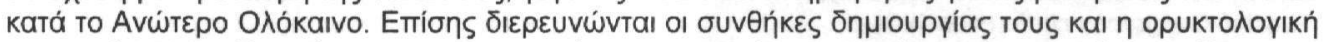

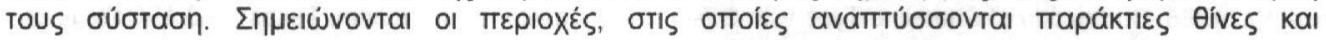

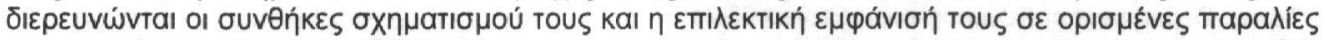

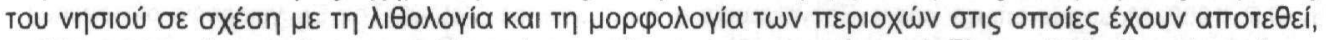

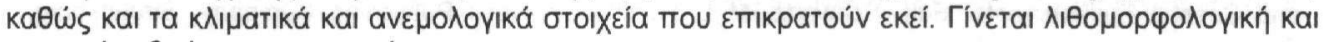

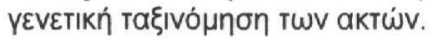

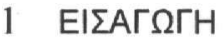

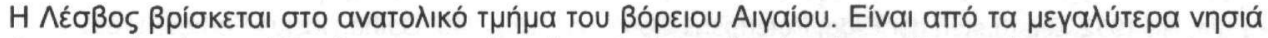

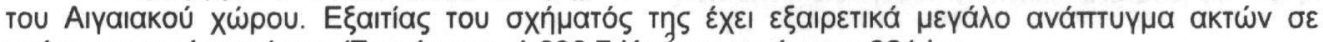

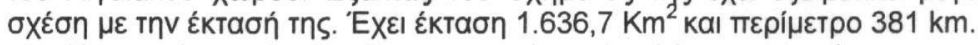

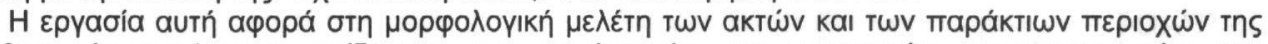

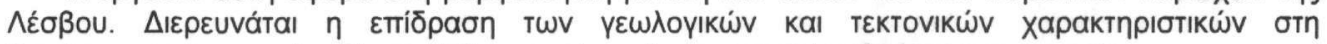

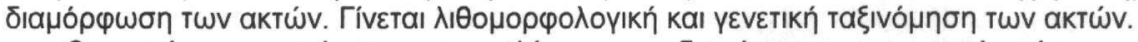

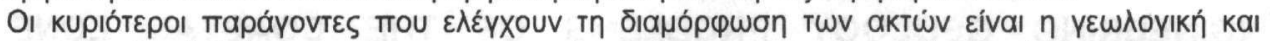

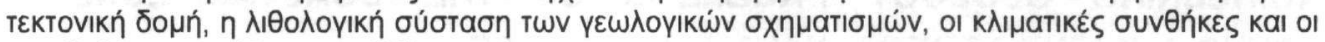

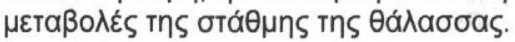

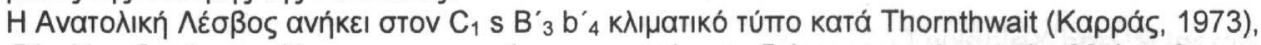

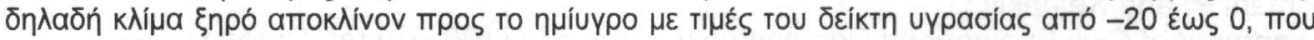

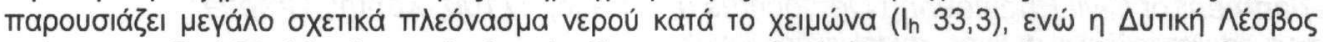

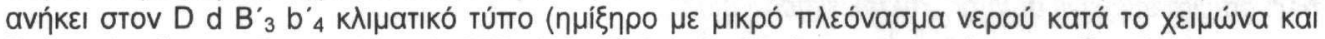

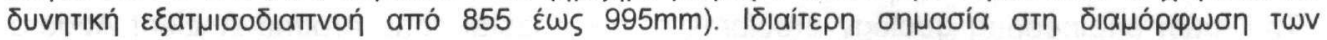

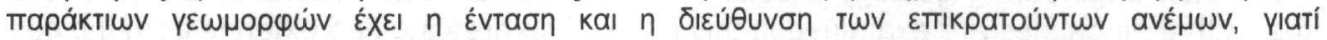

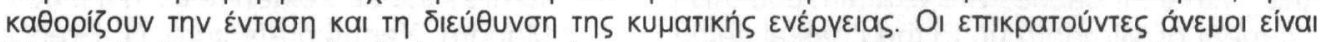

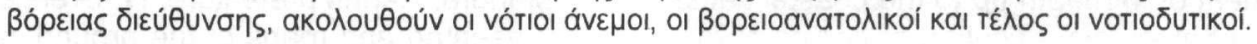

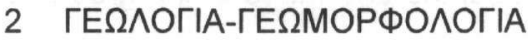

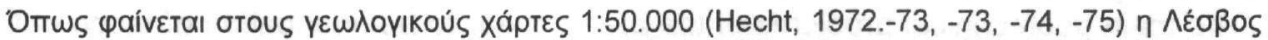

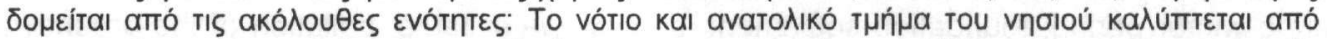

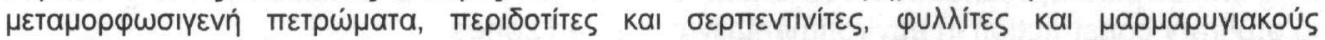

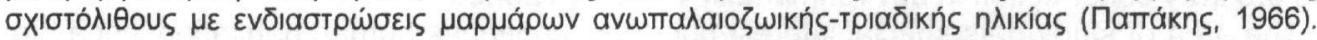

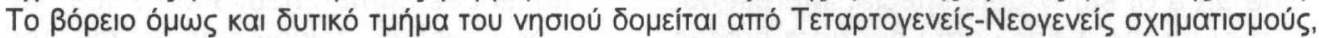

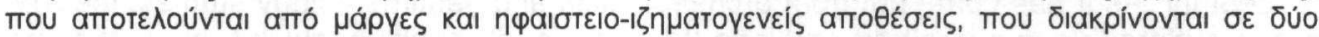

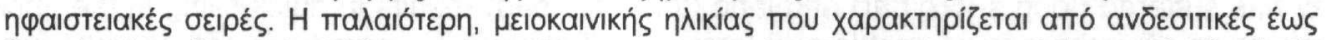

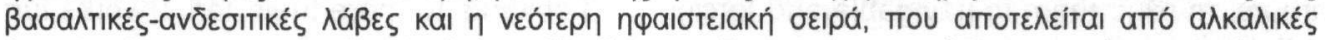

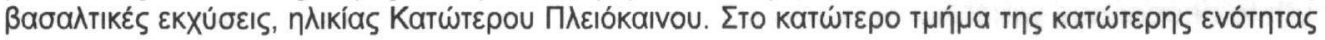




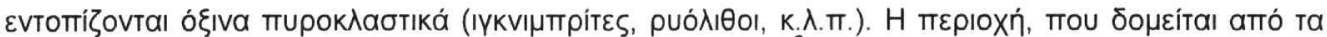

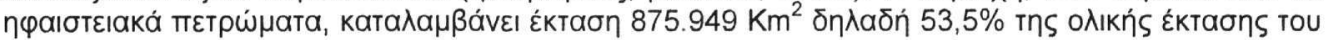
vฤซıú.

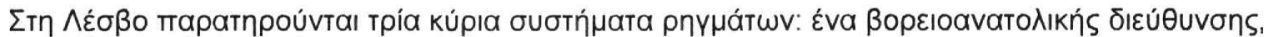

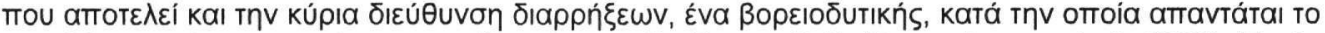

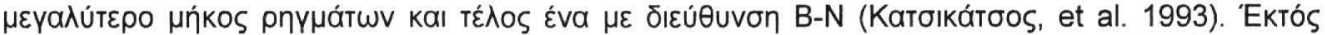

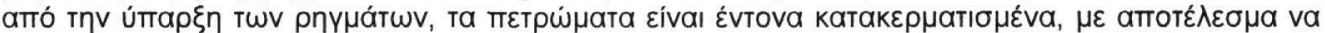

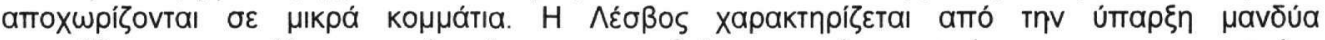

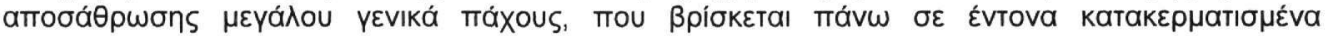

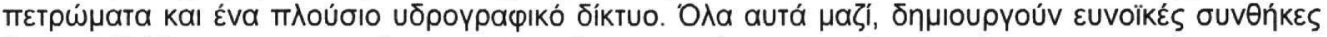

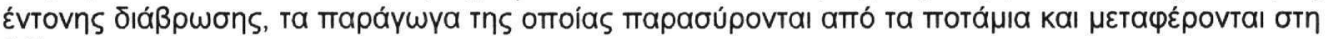

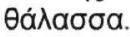

\section{2 ПАРАKTIE $\Sigma$ MOPФЕ $\Sigma$ AПOӨE $\Sigma \mathrm{H} \Sigma$}

\subsection{Beach-rocks}

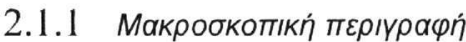

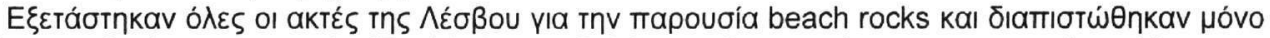

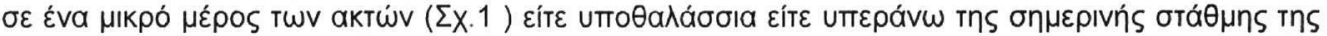

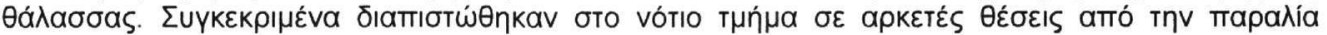

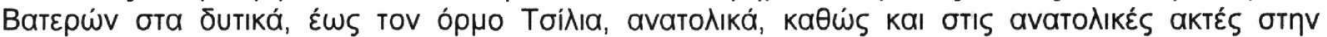

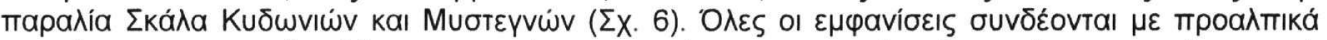

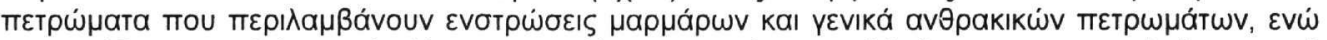

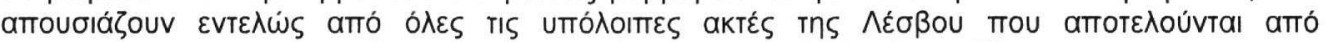

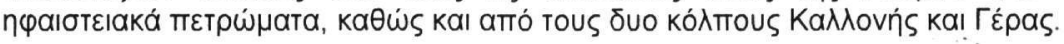

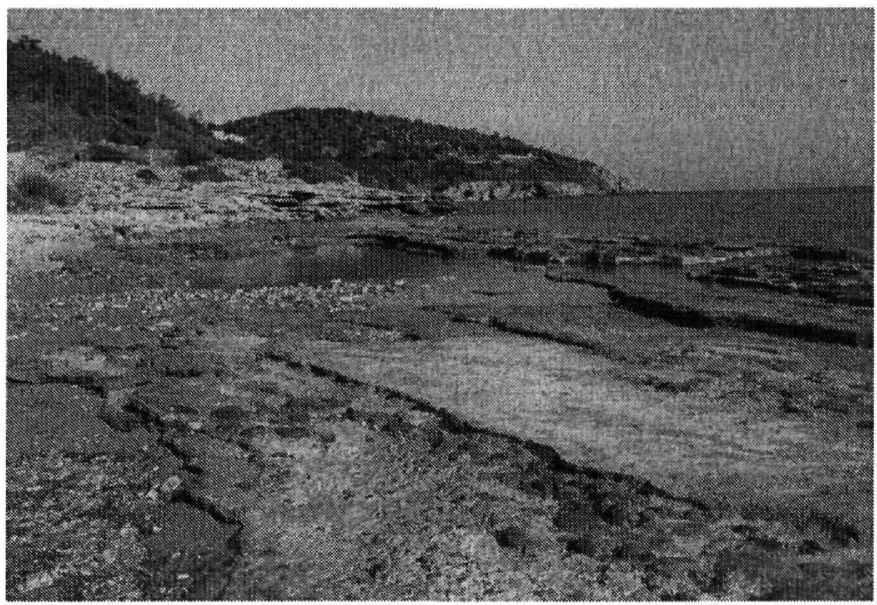

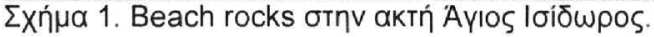

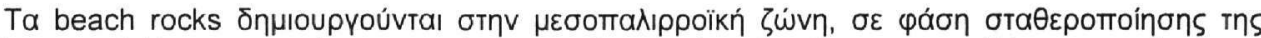

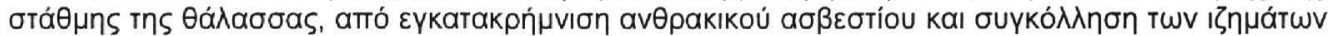
T⿰氵

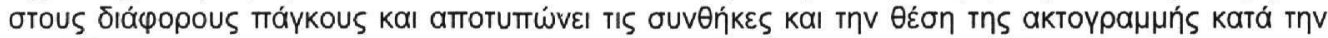

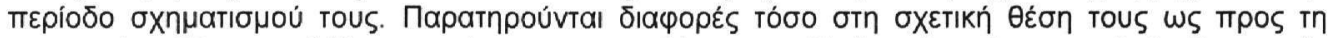

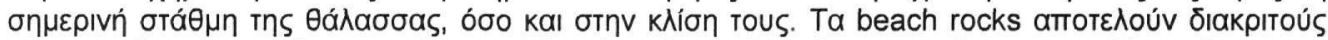

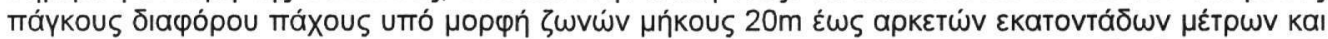

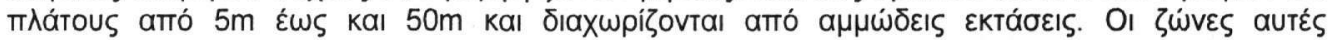

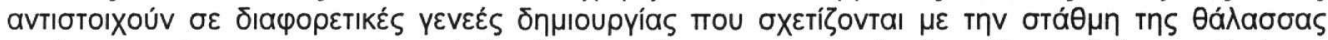

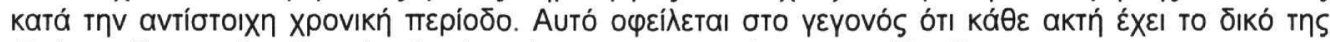

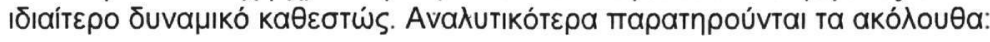




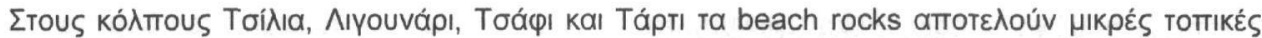

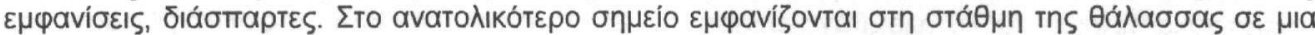

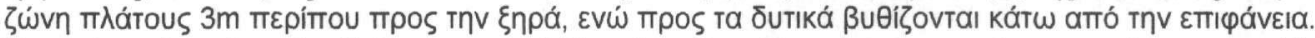

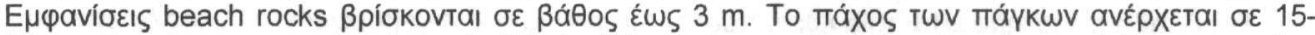

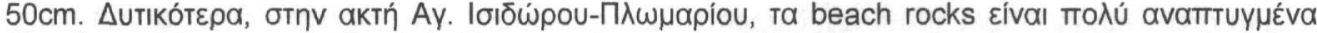

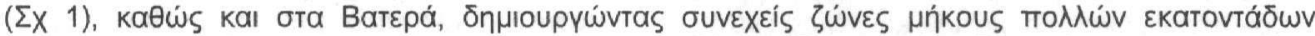

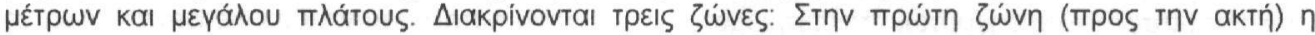

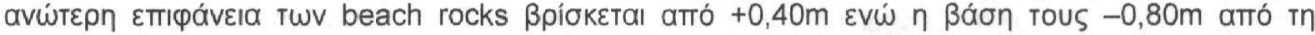

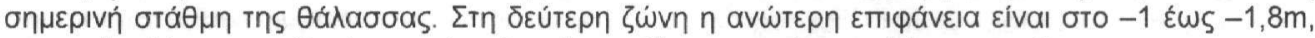

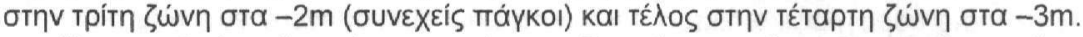

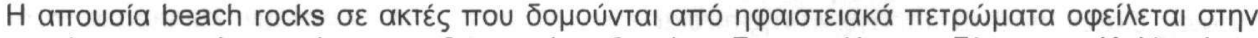

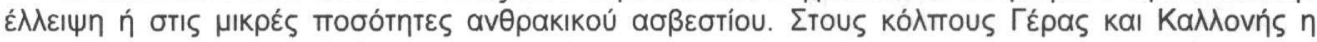

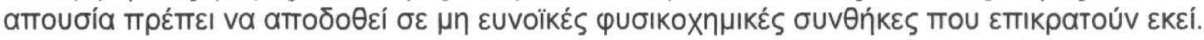

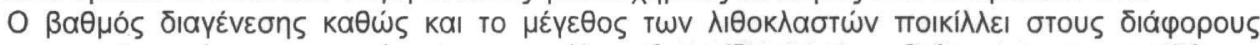

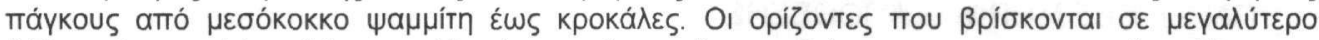

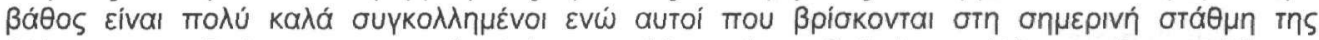

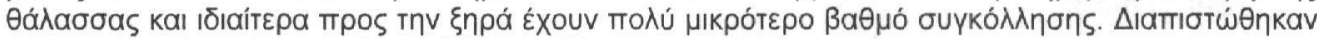

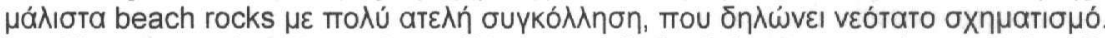

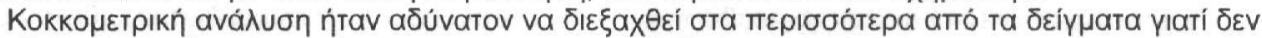

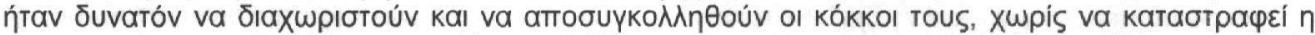

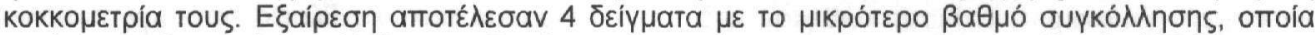

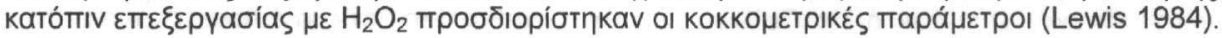

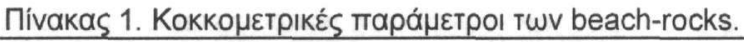

\begin{tabular}{|c|c|c|c|c|}
\hline 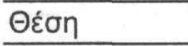 & $\mathrm{Mz}(\mathrm{mm})$ & $\sigma_{1}\left(\delta \_\alpha \beta a ́ \theta \mu ı \sigma \eta\right)$ & 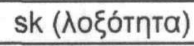 & 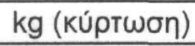 \\
\hline 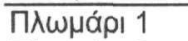 & 2,02 & 0,45(кa入ń) & 0,24 & 0.13 \\
\hline 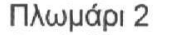 & 2,45 & 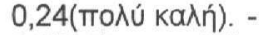 & 0,08 & 1,11 \\
\hline Aү. loíסwpos & 2,93 & 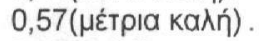 & $-0,13$ & 0,72 \\
\hline Tбíגıа & 1,87 & $0,47(\kappa a \lambda \eta ́)$ & 0,3 & 1,44 \\
\hline
\end{tabular}

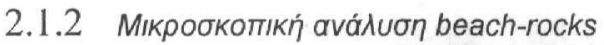

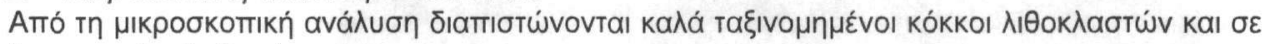

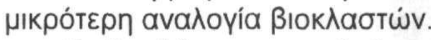

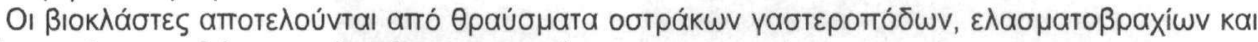

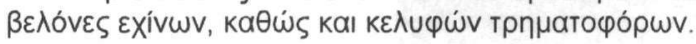

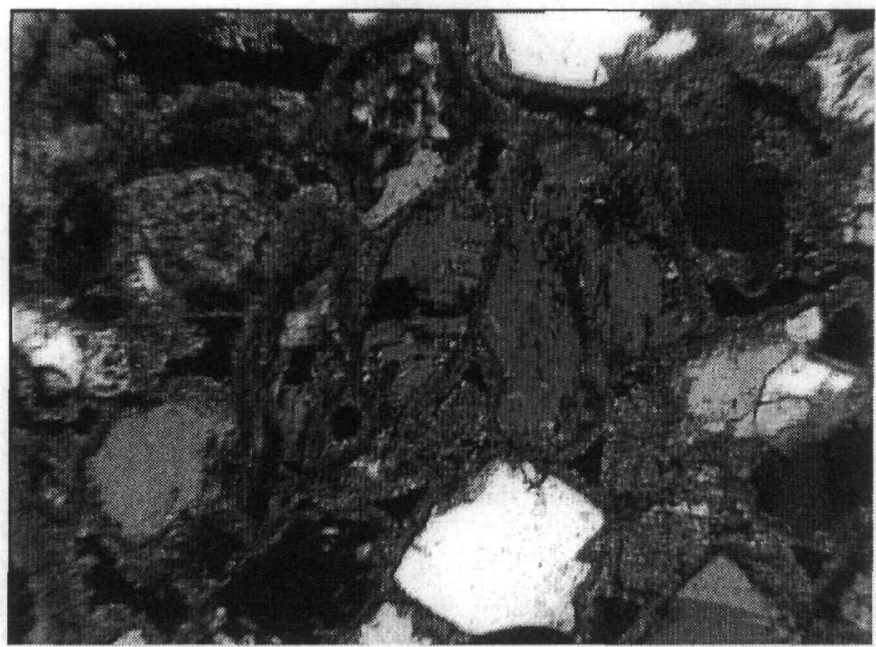

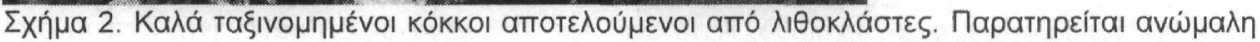

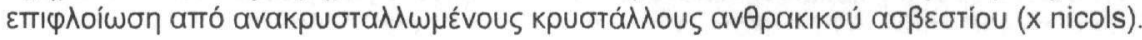




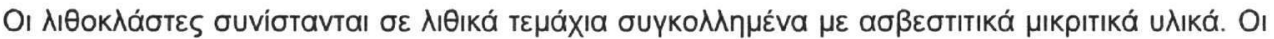

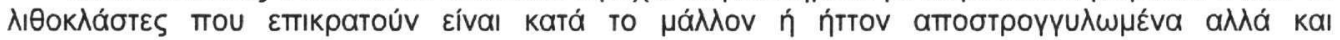

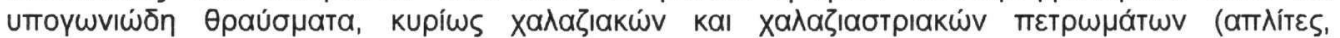

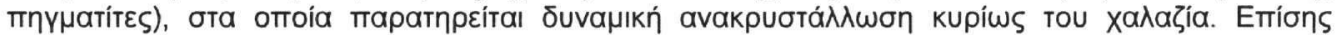

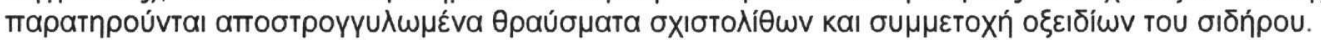

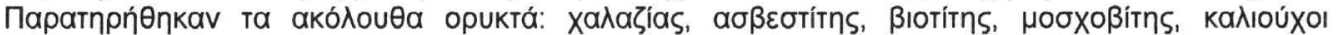

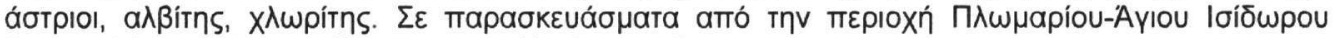

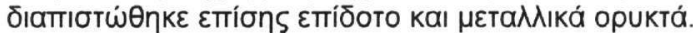

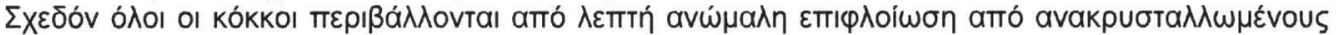

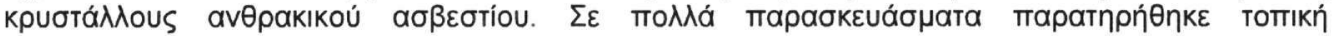

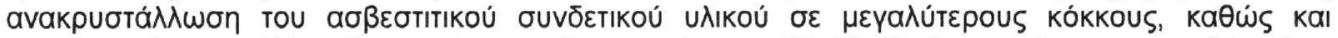

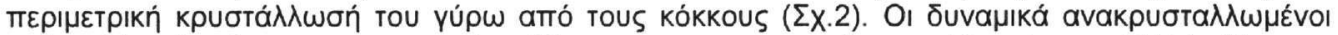

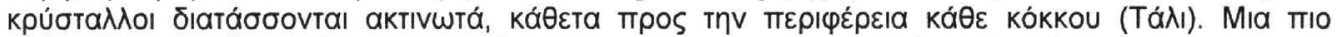

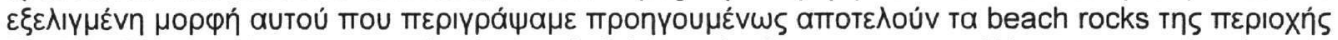

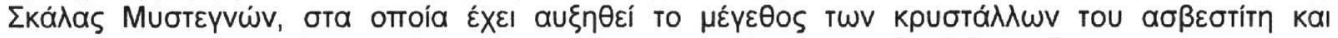

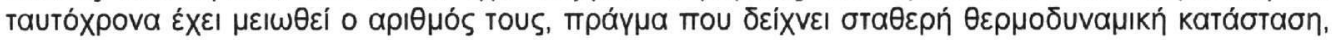

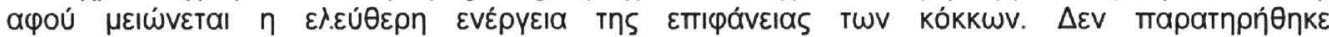

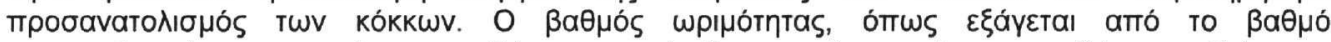

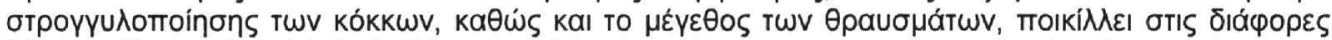

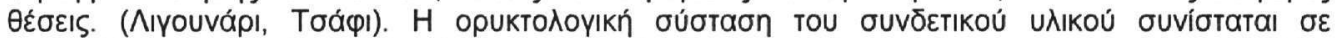

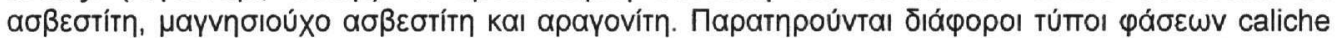

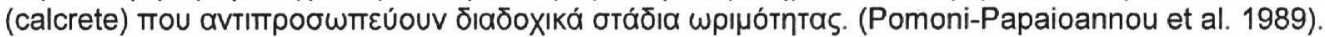

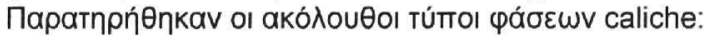

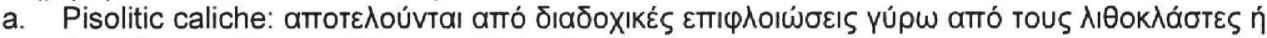

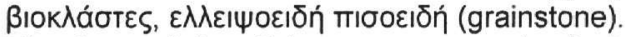

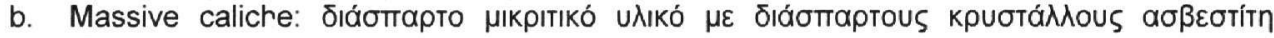
(floating texture).

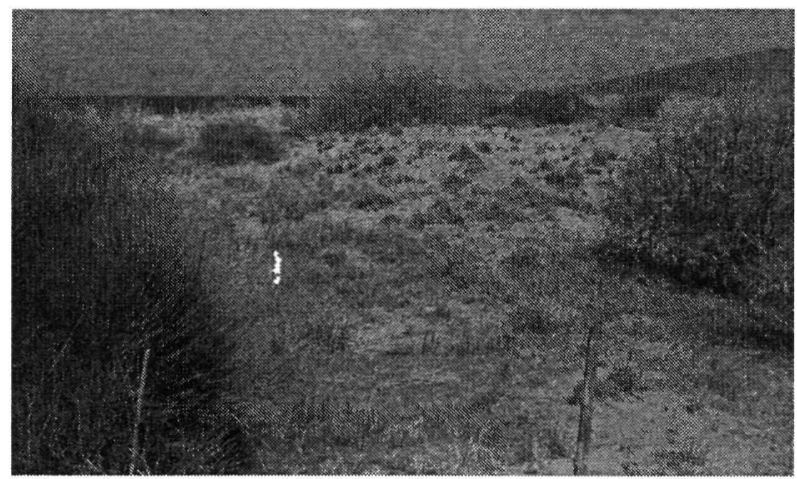

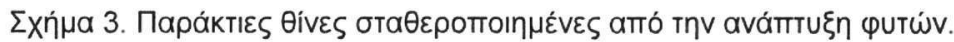

\subsection{Oives}

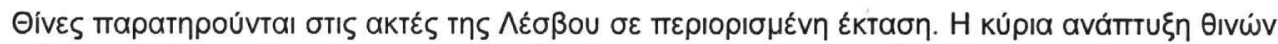

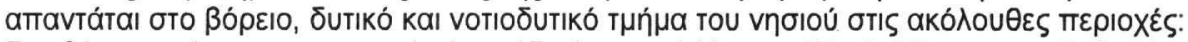

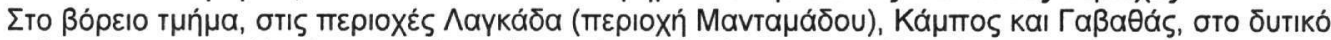

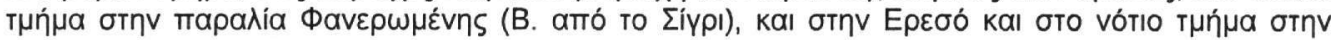

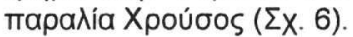

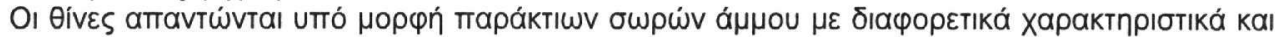

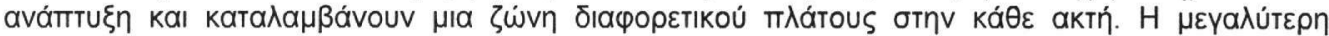

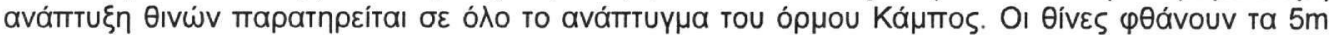

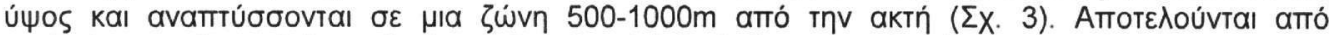

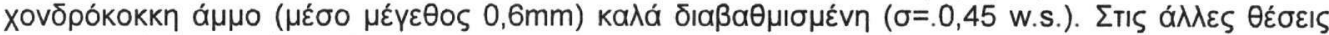

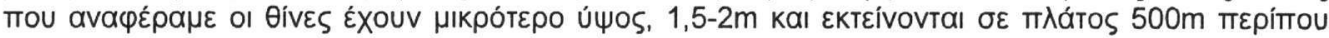

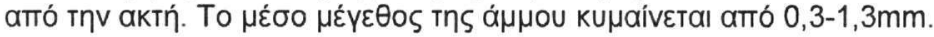




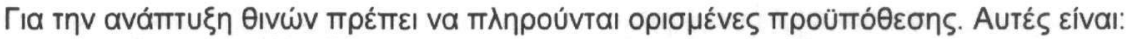

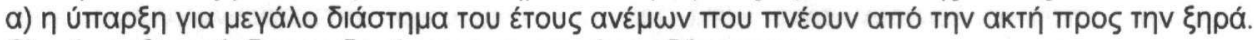

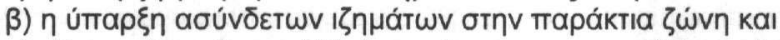

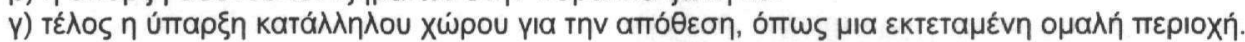

OІ пı пর́์

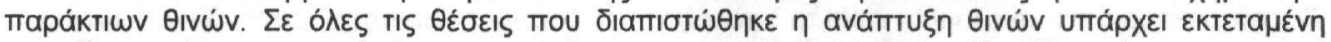

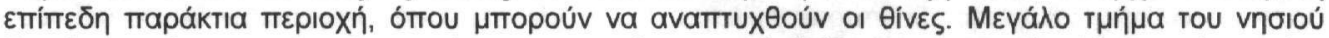

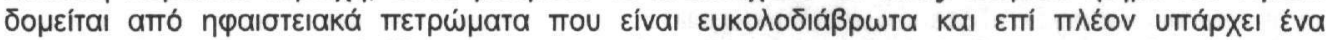

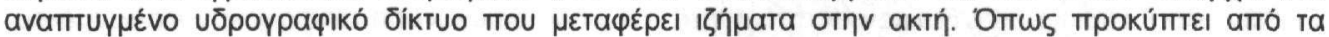

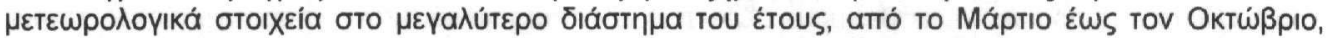

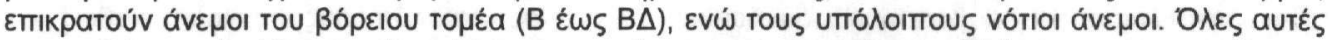

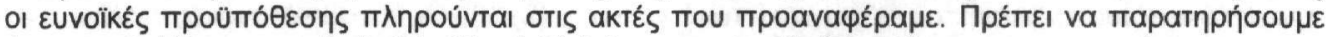

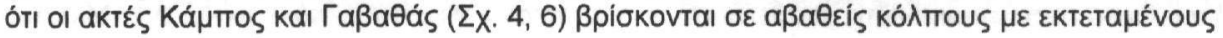
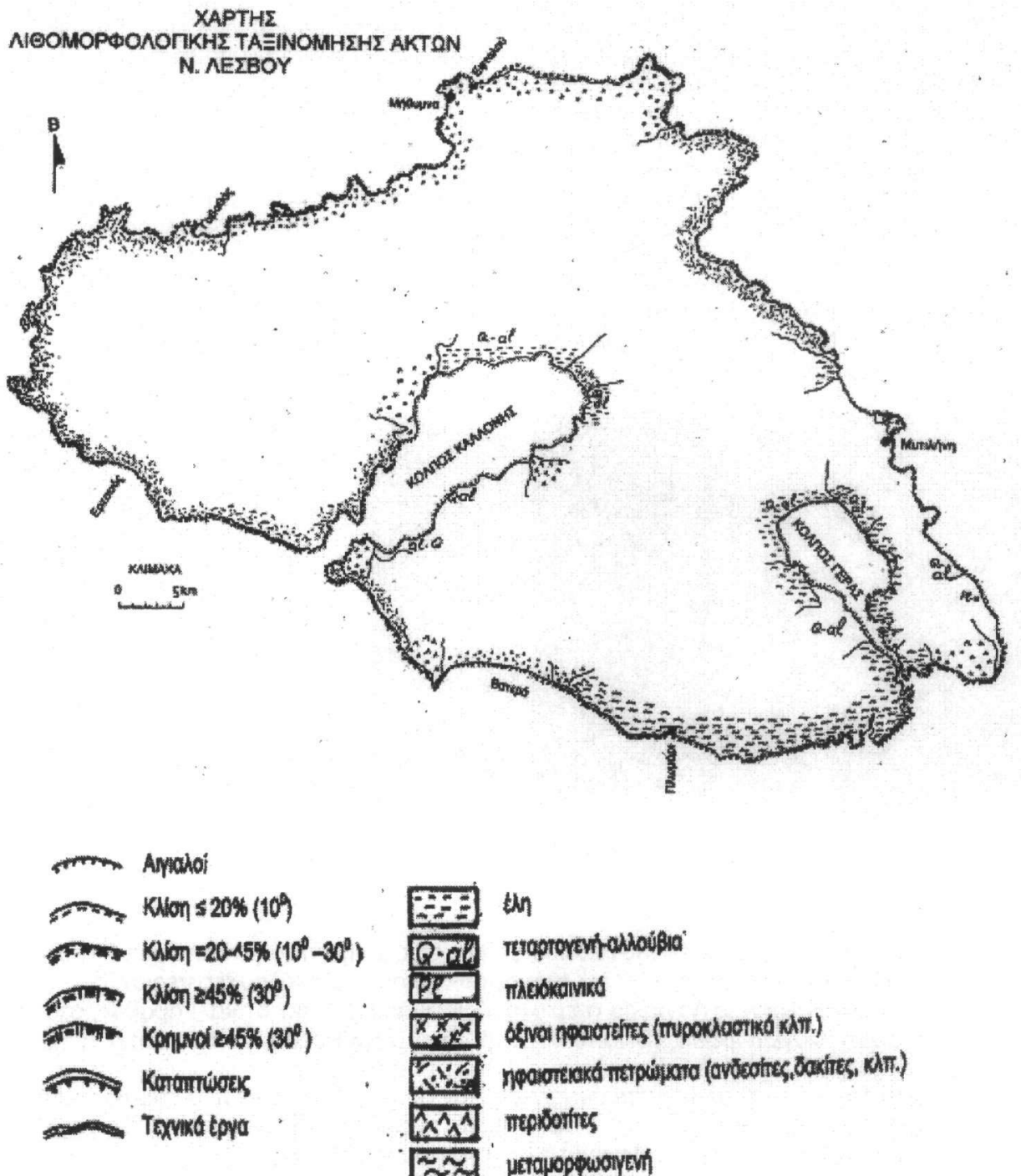

네 Tetoprovevipatioilpra" Thesokonnd

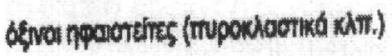

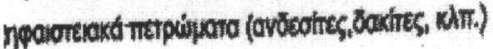
mepoüorits

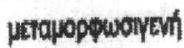

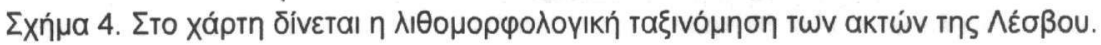




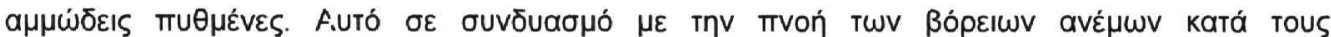

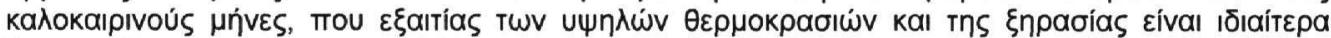

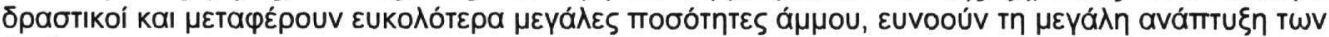
Өivúv.

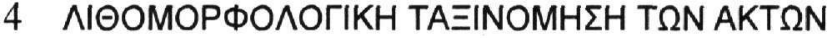

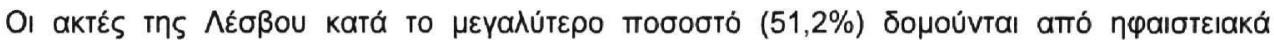

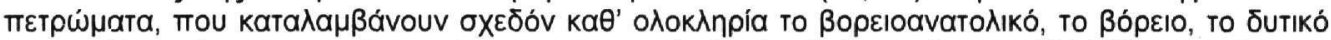

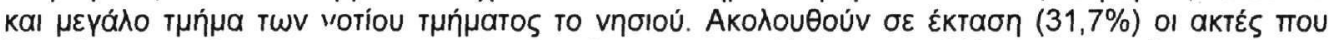

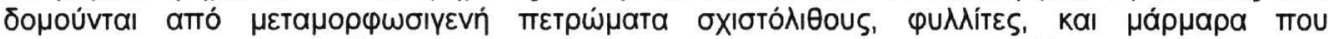

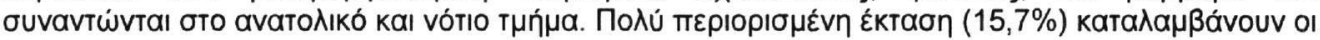

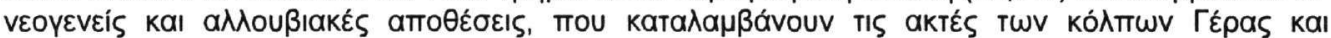

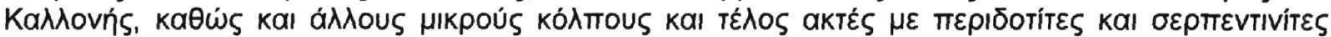

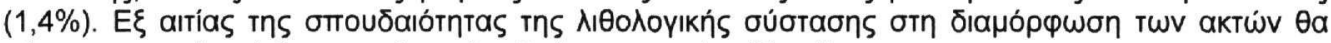

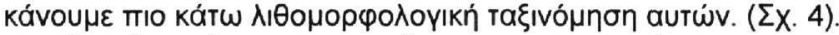

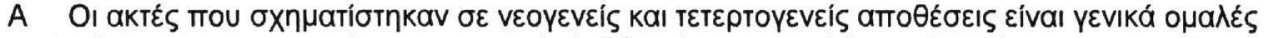

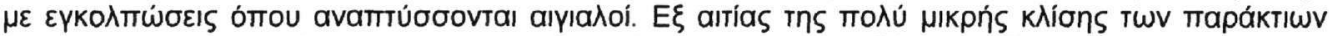

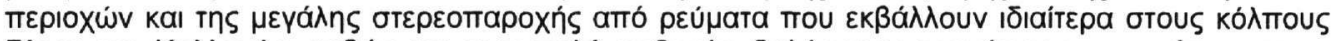

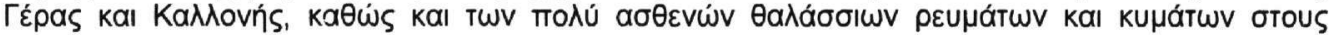

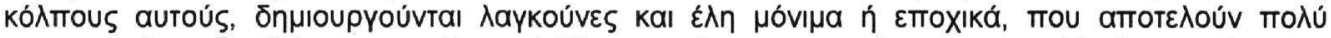

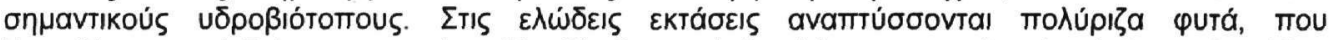

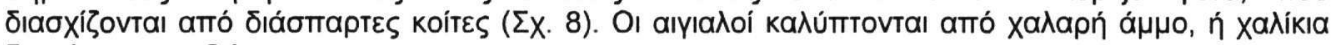

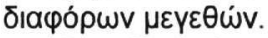

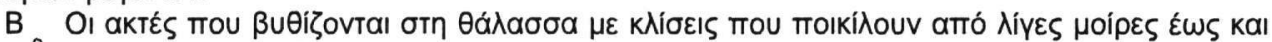

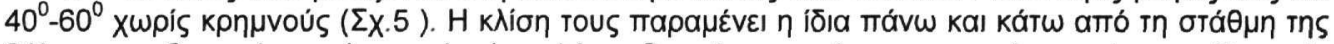

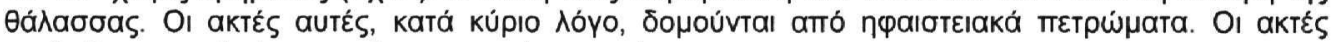

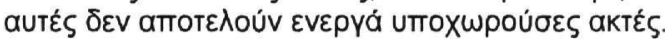

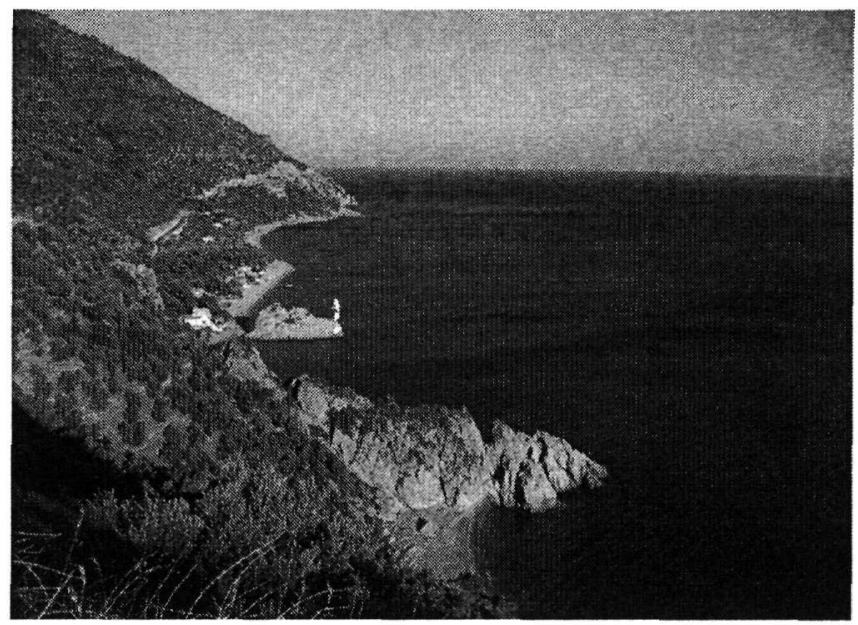

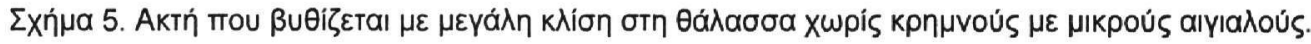

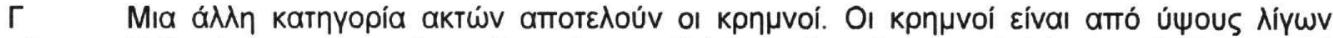

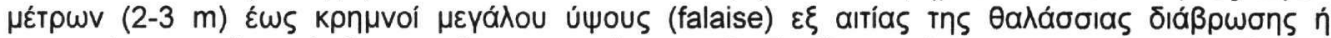

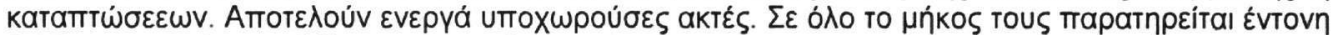

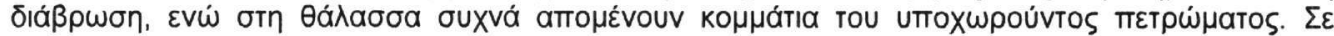

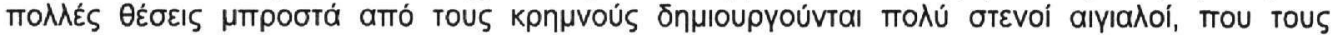

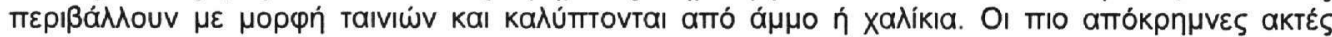

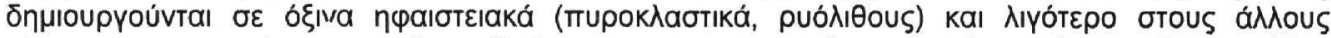

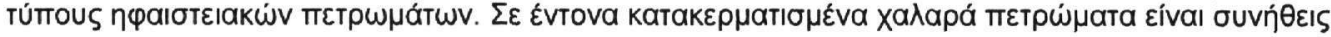




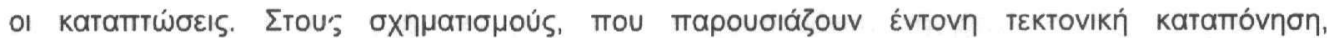

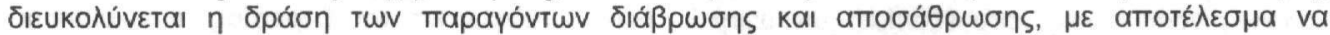

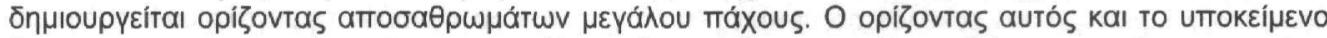

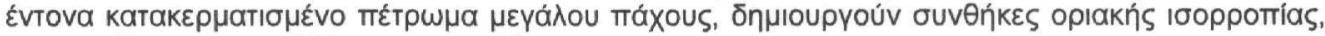

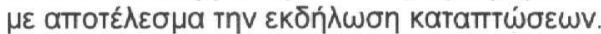

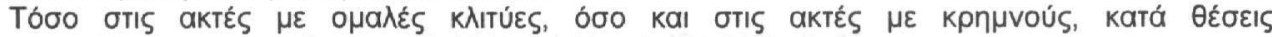

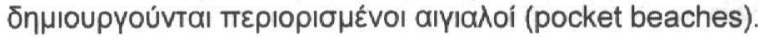

\section{5 ГENETIKH TAEINOMH $\Sigma H$ TSN AKT $\Omega N$}

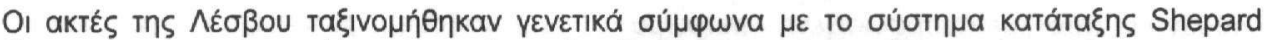

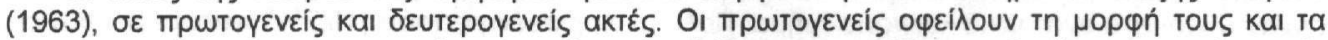

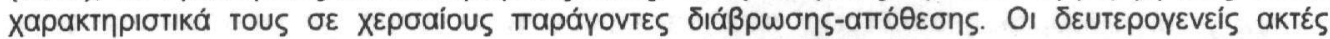

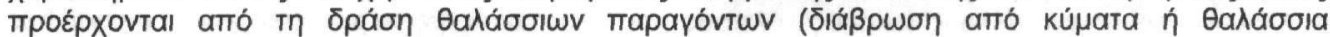

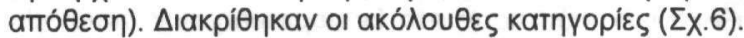

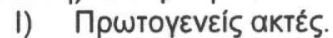

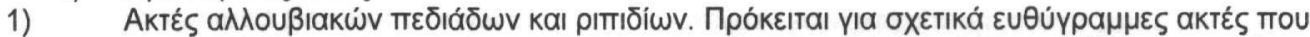

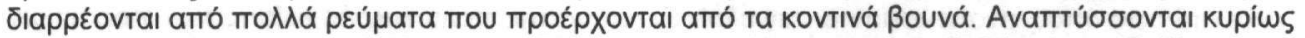

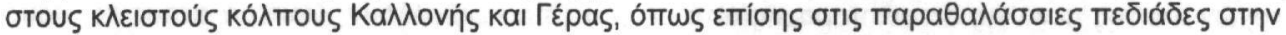

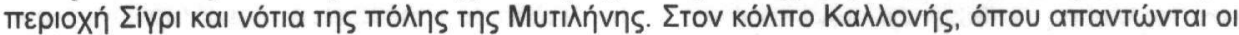
XAPTHE

TENETIKHE TAEINOMHEHE AKTSN N. AEEBOY
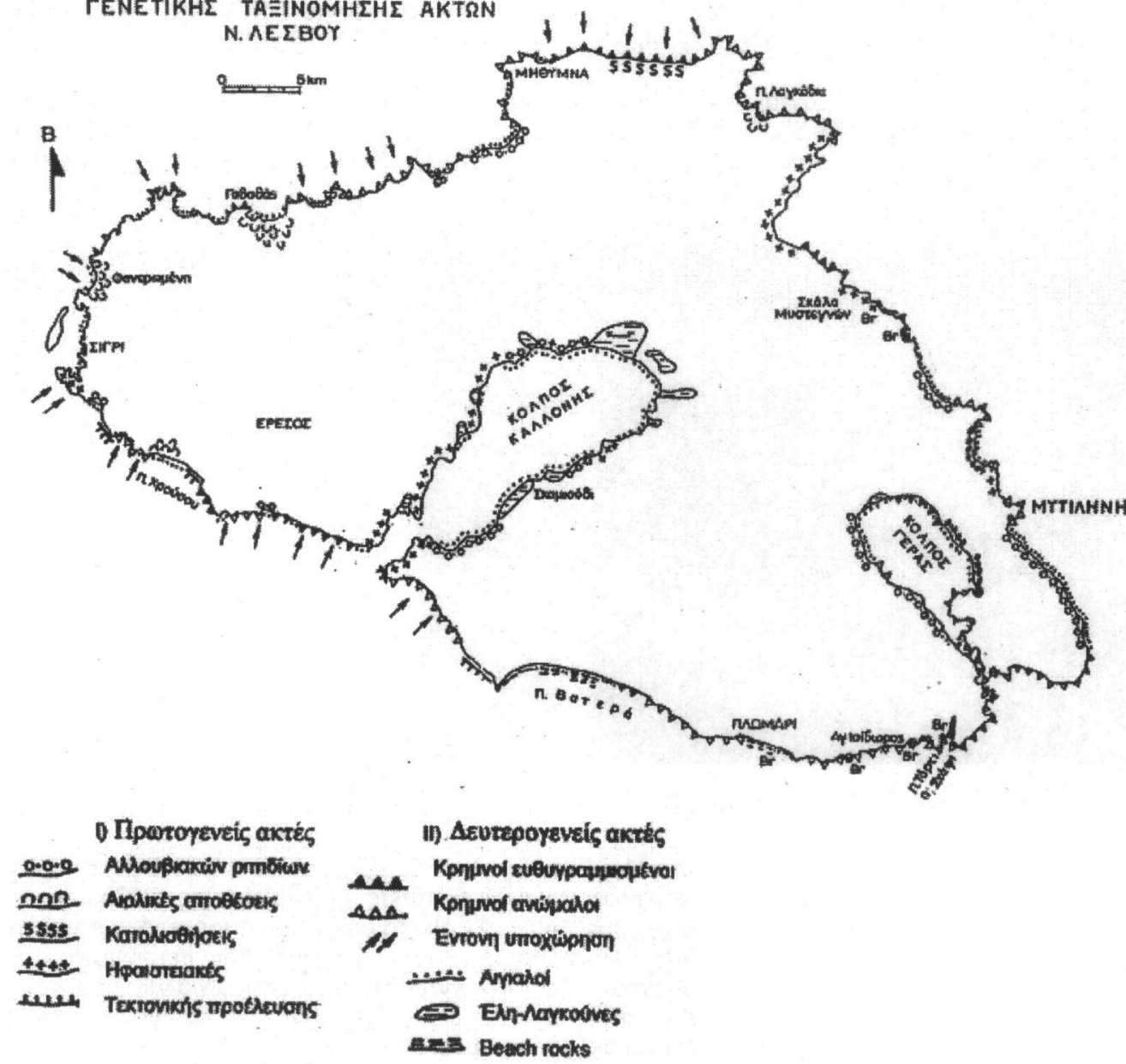

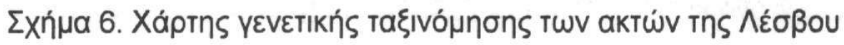




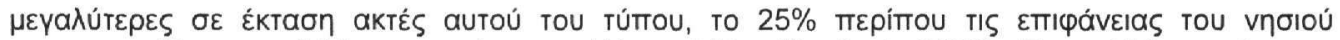

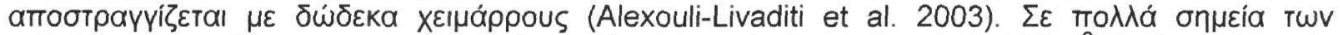

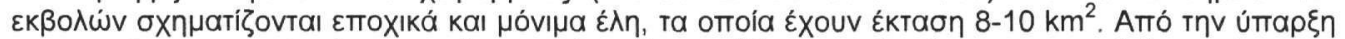

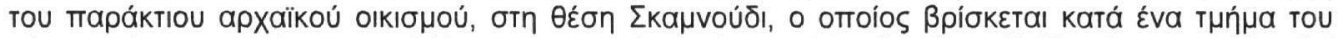

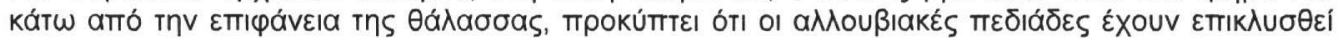

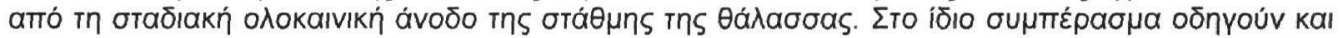

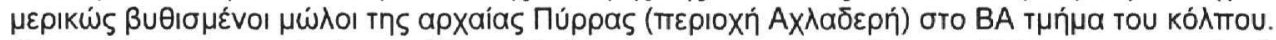

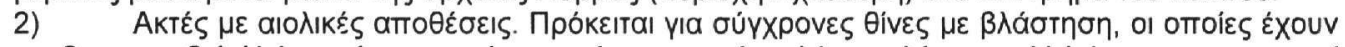

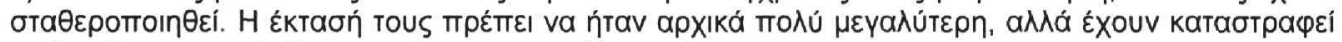

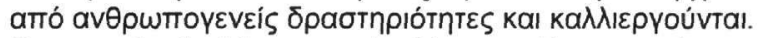

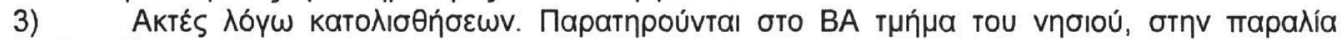

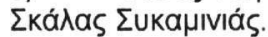

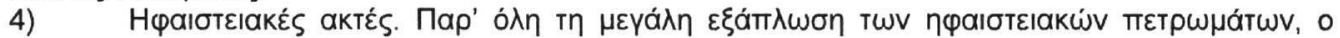

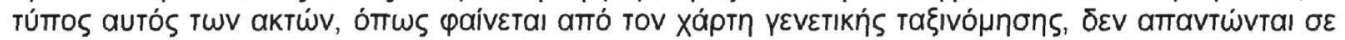

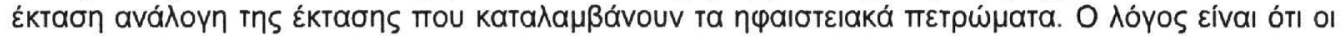

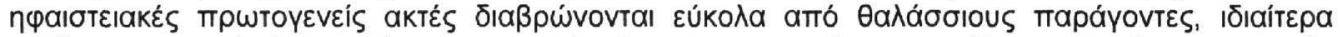

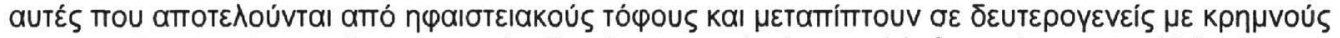

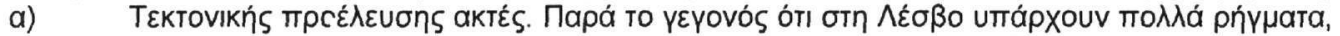

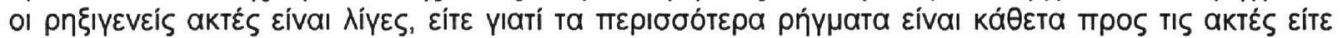

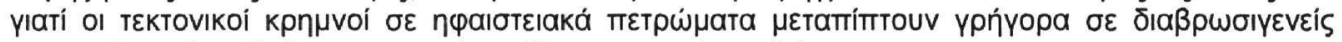

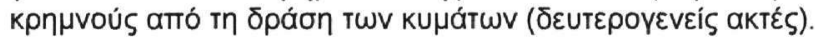

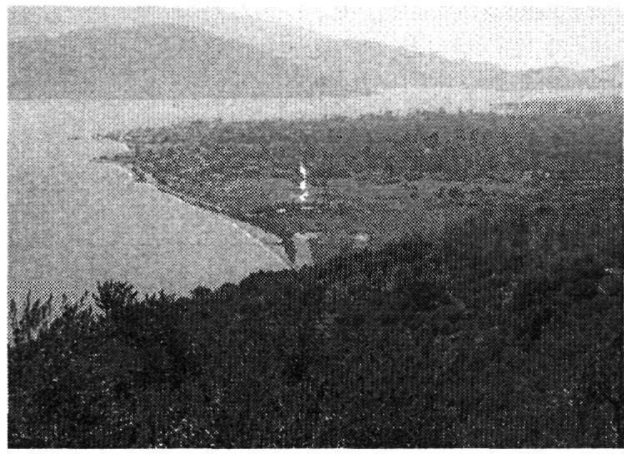

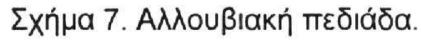

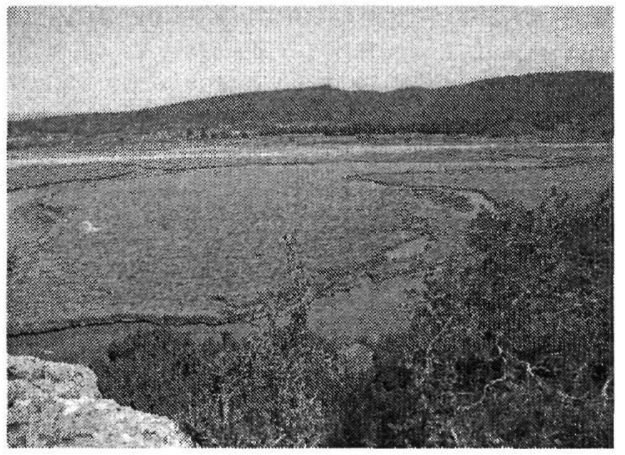

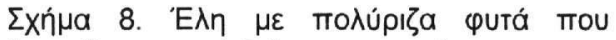

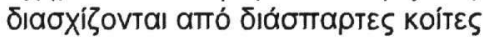

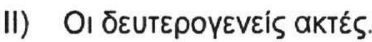

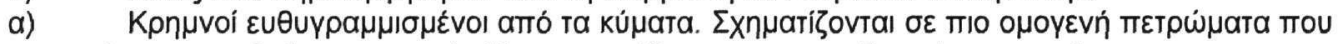

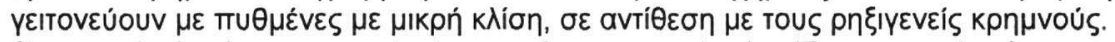

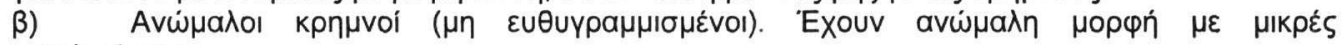

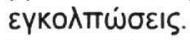

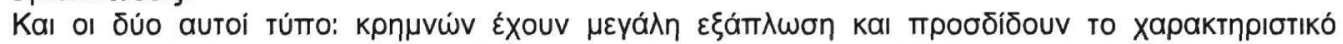

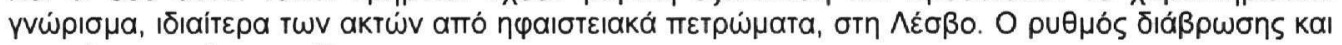

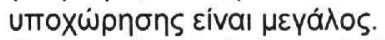

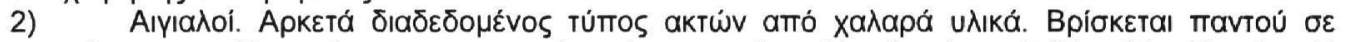

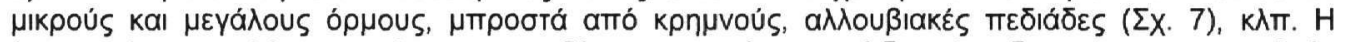

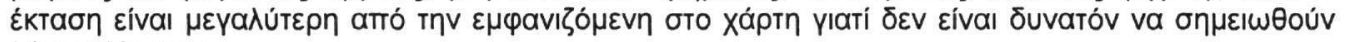

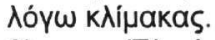

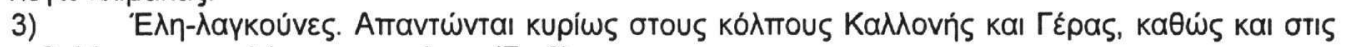

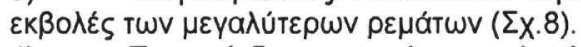

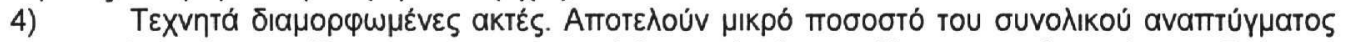

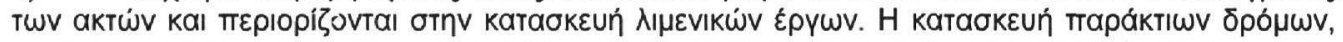

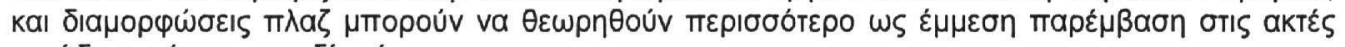

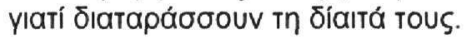




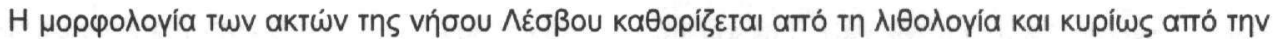

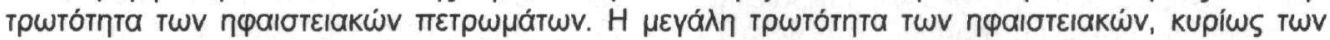

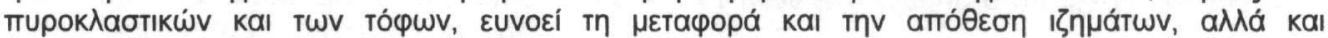

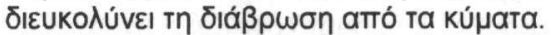

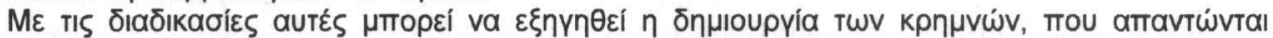

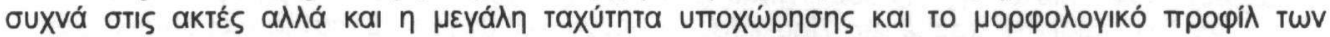

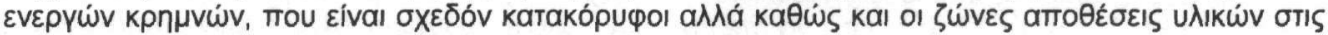

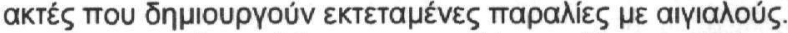

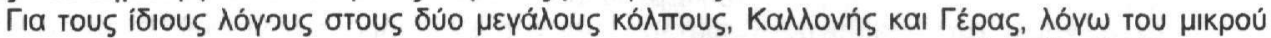

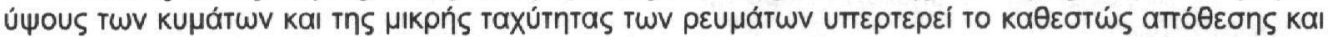

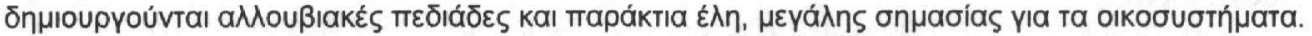

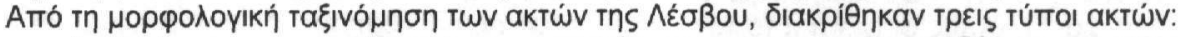

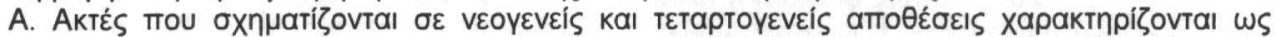

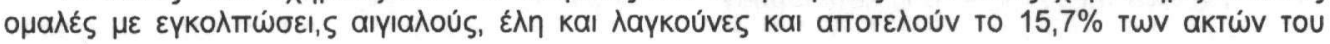
vпбıú.

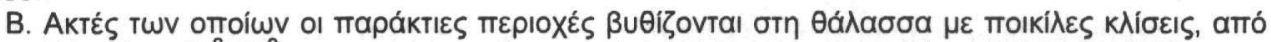

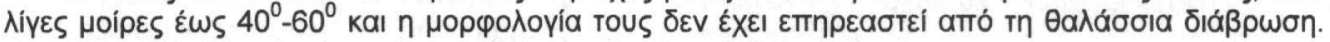

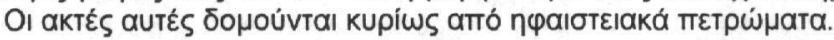

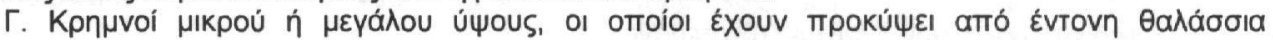

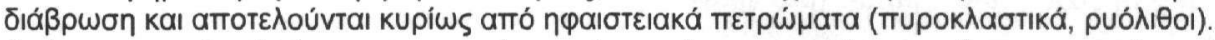

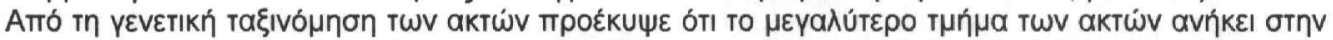

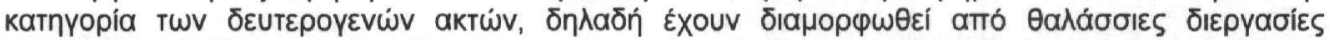

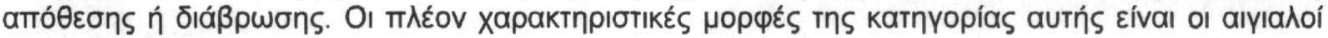

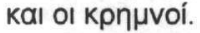

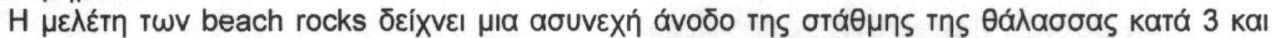

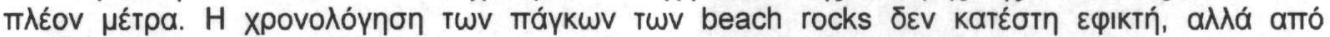

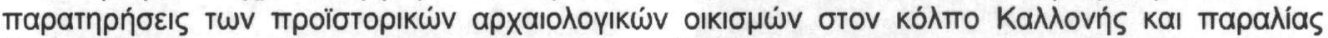

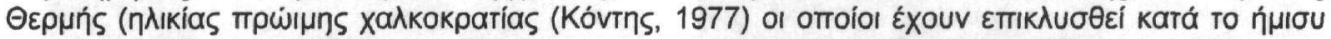

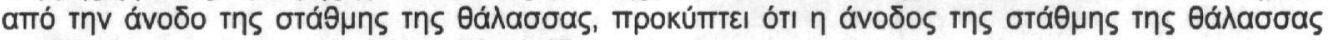

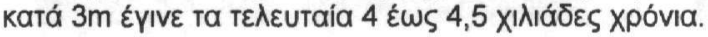

\section{ANAФOPE $\Sigma$}

Alexouli-Livaditi, A., Lykoudi E. 2003 Quantitative geomorphology study of Lesvos island drainage

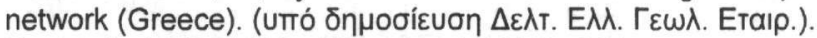

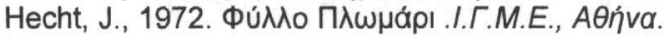

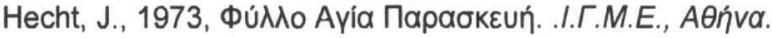

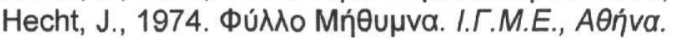

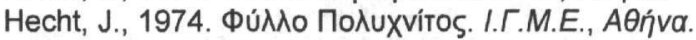

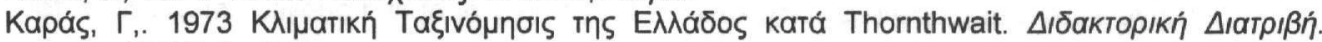

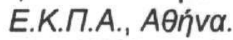

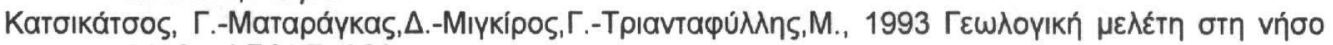

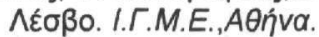

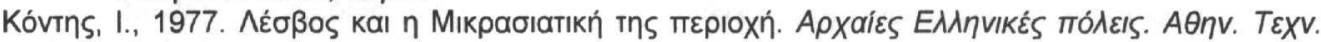
OpIAOS

Lewis, D. W., 1984. Practical Sedimentology. Stroudsburg.

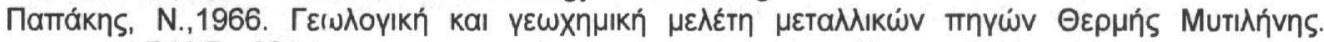

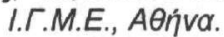

Pomoni-Papaioannou, F.\& Galeos,A. 1989. Caliche crusts in islands of Southern and Eastern Aegean and Southern Ionian Sea. Bull. Geol. Soc. Greece, VI. XXIII/1, 145-169.

Shepard, F. P., 1963. Submarine Geology. New York. (2 ${ }^{\text {nd }}$ ed). 


\title{
MORPHOLOGY OF LESVOS' ISLAND COASTS
}

\author{
Livaditis G. $^{1}$, Alexouli-Livaditi A. ${ }^{2}$ \\ ${ }^{1}$ Department of Geography-Klimatology, University of Athens, Panepistimioupoli 15784 , Athens, \\ livaditis@geo.uoa.gr \\ ${ }^{2}$ Department of Gelogical sience,National Technical University of Athens, Heroon Polytechniou, \\ Str.9, 157 27, Athens alexouli@central.ntua.gr
}

The morphology of the coasts of the island of Lesvos is controlled mainly by the erosivity of the volcanic rocks that form $>51.2 \%$ of the coasts of the island. Thus, the NE, N, W and the biggest part of the $S$ coasts consist exclusively of volcanic rocks, with the $E$ coast and the remaining part of the $S$ coast to be formed by metamorphic rocks i.e. schists, phyllites and matbles, being the $31.7 \%$ of the coast. Neogene and alluvial formations occupy the coast of the Geras and Kalloni Gulfs and secondarily some other bays representing an area of $15.7 \%$, with the remaining $1.4 \%$ of the coast to be consisted of peridotites and serpentinites.

The coasts formed by Neogene and Quaternary deposits are rather gentle incorporating small bays where beaches have been formed. Furthermore, in the case of Geras and Kalloni Gulfs lagoons and swamps (permanent and/or seasonal) have been formed due to the low gradients of the coastal zone, the increased sediment fluxes from torrents and the low-energy wave and current regime; these wetlands are of great ecological importance. Beach zone consists of sand and pebbles of varying size, whilst the backshore zone is covered by grass and is traversed by scattered creeps.

Beach rocks have been identified below or under the sea level, which are present in coasts of carbonate rocks but they are absent from those of volcanic rocks. The study of beach rocks showed a discontinuous uplifting of the sea level which reaches up to $3 \mathrm{~m}$ during the last $4000-4500$ years.

Sloping coasts of various angles (from a few degrees up to 40-60 degrees) with a rather smooth relief deep into the sea; their slope remains the same above and under the sea level. Lithologically, these coasts consist mainly of volcanic rocks. This type of coast does not belong to the category of actively retreating coasts.

Cliffs constitute another category of coasts. They vary from cliffs of few meters height $(2-3 \mathrm{~m})$ to cliffs of bigger size (falaise), being the product of marine erosion. These coasts belong to the category of actively retreating coasts. Along their coastline intensive erosion is observed; whilst in some places blocks of the retreating rocky coast remain offshore. In other places narrow shores, consisting of sand and pebbles, are formed on the foot of the cliff. The most steep coasts are formed primarily in acid volcanic rocks (pyroclastic, ryolitic) and secondarily in other type of volcanic rocks.

In the case of intensively broken cracked loose rocky formations, rockfalls are also occurred frequently. In the case of formations that undergone intensive tectonic activity, weathering and eronional processes are amplified resulting to the formation of a thick weathered horizon, which with the underlain broken rock-strata often comes into a fragile equilibrium that results to rockfalls. Finally, along both the gently sloping coasts and the cliffed coasts are formed pocket beaches.

The coasts of Lesvos are characterized genetically according to the classification system of Shepard (1963), in primary and secondary coasts.

l) Primary coasts.

1) Alluvial plain coasts

2) Wind deposition coasts.

3) a) Dunes. Sand flats form in front of dunes.

4) Landslides coasts.

5) Volcanic coasts.

6) Fault coasts.

II) Secondary coasts shaped mainly by marine agencies of erosion or deposition.

1) Coasts that were created by the erosion of waves. We distinguish:

a) Wave straitened cliffs. They are distinguished from the fault coasts because they bordered by gently inclined sea floor.

2) Made irregular by wave erosion.

3 Beaches. Enough widespread type of coasts covered by sand, gravel etc.

4 Salt Marshes and marsh grass coasts

5 Swamps and Lagoons. 